\title{
A POWER SERIES METHOD FOR SOLVING NONLINEAR BOUNDARY VALUE PROBLEMS*
}

\author{
BY \\ J. A. LEAVITT \\ Institute of Technology, University of Minnesota
}

Introduction. Power series methods have been applied successfully to nonlinear initial value problems of ordinary and partial differential equations. A demonstration of the versatility of these methods can be found in works by Richtmyer [1], Lewis [2], the author [3], [4], and others. Lewis and Richtmyer considered the supersonic flow past a blunt-nosed missile as a Cauchy problem. In order to solve the system of nonlinear equations, it was necessary to develop methods of analytic continuation and algorithms for handling the algebra necessary to determine the coefficients. For a general description of these techniques, see [4].

In this paper the author converts the two point boundary value problem to.a Cauchy problem in two independent variables, where the second variable corresponds to the unknown value of the initial derivative. A (truncated) power series solution is then found by the techniques in the above-mentioned works. The approximation is completed by finding the roots of a certain polynomial equation. In this method the differential equation is solved only once. An error estimate is given in terms of the truncation error for Lipschitz continuous functions $f$, where $f$ satisfies $u^{\prime \prime}=f\left(x, u, u^{\prime}\right)$. The results of applying this method to several nonlinear problems are presented. An appendix is included to illustrate how the algorithms may be used.

Derivation of the method. Let us consider the general boundary value problem:

$$
u^{\prime \prime}=f\left(x, u, u^{\prime}\right), \quad u\left(x_{0}\right)=a, \quad u\left(x_{1}\right)=b,
$$

and $f(x, y, z)$ is an analtyic function in each variable. There is no loss of generality if we take $x_{0}=0$. If we were to write this as a first order system, we would have

$$
\begin{aligned}
u^{\prime} & =v, & u(0) & =a ; \\
v^{\prime} & =f(x, u, v), & v(0) & =\eta+\eta_{0} .
\end{aligned}
$$

Unfortunately, we do not know the value of $\eta$ such that $u\left(x_{1}\right)=b$; usually a great deal of computational time is spent trying to estimate this value. However, we may consider that the solution depends on $\eta$ and write

$$
\begin{aligned}
u_{x}(x, \eta) & =v(x, \eta), & u(0, \eta) & =a ; \\
v_{x}(x, \eta) & =f(x, u(x, \eta), v(x, \eta)), & v(0, \eta) & =\eta+\eta_{0} .
\end{aligned}
$$

Equations (2) define a Cauchy problem in two variables. We find as a solution

$$
u(x, \eta)=\sum_{i=0}^{\infty} \sum_{j=0}^{\infty} \alpha_{i j} x^{i} \eta^{j}, \quad v(x, \eta)=\sum_{i=0}^{\infty} \sum_{j=0}^{\infty} \beta_{i j} x^{i} \eta^{j} .
$$

*Received July 17, 1967; revised version received February 4, 1968. This paper was sponsored, in part, by the National Science Foundation, Grant No. GP-4572. 
In practice, we only calculate

$$
U(x, \eta)=\sum_{i=0}^{N} \sum_{i=0}^{N} \alpha_{i j} x^{i} \eta^{i}, \quad V(x, \eta)=\sum_{i=0}^{N} \sum_{j=0}^{N} \beta_{i i} x^{i} \eta^{i} .
$$

$U(x, \eta)$, by definition, satisfies the left-hand end condition, but we still are left with the problem of resolving

$$
u\left(x_{1}\right)=U\left(x_{1}, \eta\right)=b .
$$

The solution to this problem is determined by the real roots of the polynomial equation

$$
C^{\prime}\left(x_{1}, \eta\right)=\sum_{i=0}^{N} \sum_{i=0}^{N} \alpha_{i j} x_{1}^{i} \eta^{i}=b .
$$

The approximation to the solution is then given by

$$
u(x) \simeq U\left(x, \eta_{k}\right),
$$

where the $\eta_{k}$ are the real roots of (6). In the actual computations, the real parts of the roots with small imaginary parts were also used. In terms of this formulation, the problem may not possess a real solution, and if it does, the solution may not be unique.

In Example $\mathrm{V}$ below two solutions are generated. It is impossible to tell, without some additional knowledge, which is the true solution. However, this is not a deficiency in the method, for it is not unusual for the two point boundary value problem to have several solutions, all physically meaningful. Thus this method has the capacity to generate more than one of these solutions at a time.

It may be that the solution does not converge up to the second endpoint. In that case, the following method of analytic continuation is suggested.

Let us solve again

$$
\begin{array}{ll}
U_{x}(x, \eta)=V(x, \eta), & U(0, \eta)=a, \\
V_{x}(x, \eta)=f(x, U, V), & V(0, \eta)=\eta+\eta_{0} .
\end{array}
$$

Let us assume that these solutions converge up to $x=\bar{x}$. If we let $z=x-\bar{x}$, we have the equations

$$
\begin{array}{ll}
U_{1 z}(z, \eta)=V_{1}(z, \eta), & U_{1}(0, \eta)=U(\bar{x}, \eta), \\
V_{1 z}(z, \eta)=f\left(z+\bar{x}, U_{1}, V_{1}\right), & V_{1}(0, \eta)=V(\bar{x}, \eta) .
\end{array}
$$

If the solution $U_{1}(z, \eta)$ converges up to $z=x_{1}-\bar{x}$, then we may use the same procedure and find the real roots $\eta_{k}$; otherwise the process may be repeated.

A further possibility is that Eq. (1) is singular at both endpoints. In such a case, we may try to expand the solution about the midpoint $z_{0}=\left(x_{0}+x_{1}\right) / 2$ by using the change of variables $z=x-z_{0}$. This yields the following Cauchy problem in three variables:

$$
\begin{array}{ll}
U_{z}(z, \nu, \eta)=V(z, \nu, \eta), & U(0, \nu, \eta)=\nu+\nu_{0} . \\
V_{z}(z, \nu, \eta)=f\left(z+z_{0}, U, V\right), & V(0, \nu, \eta)=\eta+\eta_{0} .
\end{array}
$$

It is necessary to solve the two equations

$$
U\left(\left(x_{0}-x_{1}\right) / 2, \nu, \eta\right)=a, \quad U\left(\left(x_{1}-x_{0}\right) / 2, \nu, \eta\right)=b
$$


which is, in general, very difficult. However, the pairs of real solutions to $(10),\left(\nu_{k}, \eta_{k}\right)$, will yield the approximations to $u(x)$.

We may apply the same technique to systems of equations by adding one parameter for each equation.

Error estimates. In order to estimate $|u(x)-U(x)|$, let us consider the problem $w(x)=u(x)-U(x), \quad w(0)=w(1)=0, \quad w^{\prime \prime}(x)=f\left(x, u, u^{\prime}\right)-\bar{f}\left(x, U, U^{\prime}\right)$,

where

$$
U^{\prime \prime}(x)=\bar{f}\left(x, U, U^{\prime}\right)=f\left(x, U, U^{\prime}\right)+\epsilon(x) .
$$

$\epsilon(x)$ is the truncation term and depends on $\eta$. The Green's solution to this problem is

$$
\begin{aligned}
w(x)=(x-1) \int_{0}^{x} t\left(f\left(t, u, u^{\prime}\right)-\bar{f}\left(t, U, U^{\prime}\right)\right) d t & \\
& \quad-x \int_{x}^{1}(1-t)\left(f\left(t, u, u^{\prime}\right)-\bar{f}\left(t, U, U^{\prime}\right)\right) d t .
\end{aligned}
$$

We shall assume that $f(x, y, z)$ is Lipschitz continuous in $y$ and $z$ with constant $K$, i.e.,

$$
\left|f\left(x, y_{1}, z_{1}\right)-f\left(x, y_{2}, z_{2}\right)\right| \leq K\left\{\left|y_{1}-y_{2}\right|+\left|z_{1}-z_{2}\right|\right\} \text {. }
$$

We find by differentiating (12)

$w^{\prime}(x)=\int_{0}^{x} t\left(f\left(t, u, u^{\prime}\right)-\bar{f}\left(t, U, U^{\prime}\right)\right) d t-\int_{x}^{1}(1-t)\left(f\left(t, u, u^{\prime}\right)-\bar{f}\left(t, U, U^{\prime}\right)\right) d t$.

Using the definition of $\bar{f}\left(x, U, U^{\prime}\right)$ and Iipschitz continuity we find

$$
\begin{aligned}
|w(x)| \leq & K(1-x) \int_{0}^{x} t\left\{|w(t)|+\left|w^{\prime}(t)\right|\right\} d t \\
& +K x \int_{x}^{1}(1-t)\left\{|w(t)|+\left|w^{\prime}(t)\right|\right\} d t+E_{0}(x) . \\
\left|w^{\prime}(x)\right| \leq & K \int_{0}^{x} t\left\{|w(t)|+\left|w^{\prime}(t)\right|\right\} d t \\
& +K \int_{x}^{1}(1-t)\left\{|w(t)|+\left|w^{\prime}(t)\right|\right\} d t+E_{1}^{\prime}(x),
\end{aligned}
$$

where

$$
\begin{aligned}
& E_{0}(x)=(1-x)\left|\int_{0}^{x} t \varepsilon(t) d t\right|+x\left|\int_{x}^{1}(1-t) \varepsilon(t) d t\right| \geq 0, \\
& E_{1}(x)=\left|\int_{0}^{x} t \varepsilon(t) d t\right|+\left|\int_{x}^{1}(1-t) \varepsilon(t) d t\right| \geq 0,
\end{aligned}
$$

and $0 \leq x \leq 1$. Let $W=\max _{0 \leq x \leq 1}\left\{|w|+\left|w^{\prime}\right|\right\}$; then

$$
\begin{aligned}
& |w(x)| \leq K W(1-x) \int_{0}^{x} t d t+K W x \int_{x}^{1}(1-t) d t+E_{0}(x), \\
& \left|w^{\prime}(x)\right| \leq K W \int_{0}^{x} t d t+K W \int_{x}^{1}(1-t) d t+E_{1}(x) .
\end{aligned}
$$


Adding these two equations we obtain the inequality

$$
|w(x)|+\left|w^{\prime}(x)\right| \leq K W\left\{(2-x) \int_{0}^{x} t d t+(1+x) \int_{x}^{1}(1-t) d t\right\}+E_{0}(x)+E_{1}(x)
$$

or

$$
\begin{aligned}
|w(x)|+\left|w^{\prime}(x)\right| & \leq K W\left(\left(1-x+x^{2}\right) / 2\right)+E_{0}(x)+E_{1}(x) \\
& \leq K W / 2+E,
\end{aligned}
$$

where $E=\max _{0 \leq x \leq 1}\left\{E_{0}(x)+E_{1}(x)\right\}$.

This last inequality is true for all $x$ in the interval, so we may write

$$
W \leq K W / 2+E \text {, }
$$

which yields

$$
W \leq E /(1-K / 2), \quad K<2 .
$$

Substituting this result back into (15) gives

or

$$
|w(x)| \leq K E /(1-K / 2)\left\{(1-x) \int_{0}^{x} t d t+x \int_{x}^{1}(1-t) d t\right\}+E_{0}(x) .
$$

$$
|u(x)-U(x)|=|w(x)| \leq K E /(1-K) / 2)(x(1-x)) / 2+E_{0}(x) .
$$

Thus we have found

$$
\begin{aligned}
& |u(x)-U(x)| \\
& \leq \frac{K}{2-K} x(1-x) \max _{0 \leq x \leq 1}\left\{(2-x)\left|\int_{0}^{x} t \varepsilon(t) d t\right|+(1+x)\left|\int_{x}^{1}(1-t) \varepsilon(t) d t\right|\right\} \\
& \quad \quad+(1-x)\left|\int_{0}^{x} t \varepsilon(t) d t\right|+x\left|\int_{x}^{1}(1-t) \varepsilon(t) d t\right|, \quad 0 \leq x \leq 1 .
\end{aligned}
$$

This expression vanishes at both endpoints and is small if the truncation term, $\epsilon(x)=$ $\epsilon(x, \eta), 0 \leq x \leq 1$, is small.

Examples. Several problems were tried on the computer. They exhibit, to some extent, both the power and limitations of this method. In all the examples, except VI, $\eta_{0}=0$.

I. The following linear problem

$$
u^{\prime \prime}=u^{\prime}+2 u, \quad u(0)=2, \quad u(1)=e^{2}+e^{-1}
$$

has the solution $u(x)=e^{2 x}+e^{-x}$.

The program took $3 / 60$ seconds to calculate the coefficients of the polynomial to degree 15 and $3 / 60$ seconds to find the unique root for the first derivative:

$$
u^{\prime}(0)=U^{\prime}(0)=\eta=1 .
$$

The entire program took only $6 \frac{26}{60}$ seconds after compilation. The results are shown in Table I. In linear problems the expansion remains linear in terms of the parameter $\eta$.

II. The simple nonlinear problem

$$
u^{\prime \prime}=u u^{\prime}, \quad u(0)=1, \quad u(1)=2
$$


also yielded a good approximation. The approximate solution

$$
U(x)=\sum_{i=0}^{29} \sum_{i=0}^{29} a_{i j} x^{i} \eta^{i}
$$

produced three real roots for $\eta$ :

$$
.500,000,000,8, \quad-10.652,715,21, \quad-99.723,656,90 \text {. }
$$

This program required $27 \frac{28}{60}$ seconds to calculate the coefficients and $3 \frac{38}{60}$ seconds to find the roots.

The solution corresponding to the root $-10.652,715,21$ does not represent a convergent solution. This was determined by considering the $n$th root test of the resulting series. The numbers in the fourth column indicate that the series corresponding to the third root does not converge in the interval.

The entire program took less than 61 seconds after compilation.

The solutions to the next two problems were expanded as series about each of the endpoints, $x=0$ and $x=1$. In both cases one series about one endpoint converged considerably faster than the other. This result coincides with the convergence of the respective Taylor series expansions of the exact solutions about the different endpoints. Analytic continuation, applied to these examples, would have produced more accurate results than the solutions about the left-hand endpoint.

III. (a) In the first problem

$$
u^{\prime \prime}+\left(u^{\prime}\right)^{2}=0, \quad u(0)=1, \quad u(1)=1+\log 2,
$$

the approximate solution

$$
U(x)=\sum_{i=0}^{35} \sum_{i=0}^{35} a_{i i} x^{i} \eta^{i}
$$

produced one real root, $U^{\prime}(0)=\eta=.98411$.

This program required $35 \frac{5}{60}$ seconds to calculate the coefficients and $9 \frac{59}{60}$ seconds to find the roots. The whole program took 48 seconds after compilation.

(b) The other expansion

$$
U(x)=\sum_{i=0}^{27} \sum_{i=0}^{27} a_{i j}(1-x)^{i} \eta^{i}
$$

produced one real root $U^{\prime}(1)=\eta=-.500,000,000,1$.

The program required $13 \frac{7}{60}$ seconds to calculate the coefficients and $5 \frac{56}{60}$ seconds to find the roots. The entire program took $30 \frac{8}{60}$ seconds after compilation.

IV. (a) The approximations to the following problem converged a little faster:

$$
u^{\prime \prime} u+\left(u^{\prime}\right)^{2}=0, \quad u(0)=1, \quad u(1)=2^{1 / 2} .
$$

The approximate solution

$$
U(x)=\sum_{i=0}^{39} \sum_{i=0}^{39} a_{i j} x^{i} \eta^{i}
$$

produced one real root $U^{\prime}(0)=\eta=.49924$.

The program required $104 \frac{18}{60}$ seconds to calculate the coefficients and $18 \frac{35}{60}$ seconds to find the roots. The whole program took $137 \frac{19}{60}$ seconds after compilation. 
(b) The approximate solution

$$
U(x)=\sum_{j=0}^{23} \sum_{i=0}^{23} a_{i j}(1-x)^{i} \eta^{i}
$$

produced one real root, $U^{\prime}(1)=\eta=-.353,553,390,9$.

The program required $14 \frac{33}{60}$ seconds to calculate the coefficients and $4 \frac{6}{60}$ seconds to find the roots. The whole program took $29 \frac{11}{60}$ seconds after compilation.

The series in parts (b) of examples III and IV represent expansions about $x=1$ of $y=1+\log (1+x)$ and $y=(1+x)^{1 / 2}$ respectively. And so they converge more rapidly than the series of parts (a) which are expansions about $x=0$.

V. The next program was a good test of the method.

$$
\begin{gathered}
u^{\prime \prime}+3 u u^{\prime}+u^{3}=-\left(x^{3}+6 x^{2}+24 x+40\right) /\left(x^{3}-6 x^{2}+12 x-8\right) \\
u(0)=1, \quad u(1)=3 .
\end{gathered}
$$

The approximate solution

$$
U(x)=\sum_{j=0}^{32} \sum_{i=0}^{32} a_{i j} x^{i} \eta^{i}
$$

produced four real roots $\eta$ :

$$
1.000,005,557, \quad .095,490,169,29, \quad \$ .679,316,529, \quad 44.989,091,75 .
$$

The program required $70 \frac{58}{60}$ seconds to calculate the coefficients and 15 seconds to find the roots. The whole program took $132 \frac{29}{60}$ seconds after compilation.

The last two solutions in Table $\mathrm{V}$ evidently are not convergent.

The approximate solution to this problem was recalculated several times. Each time the number of terms in the summation was changed. The root 1.0 was always found. A root in the neighborhood of .095 was also found providing that the degree of the approximating polynomial was even. As the degree increased, this root decreased. For $N=38, \eta=.065$. While it is believed that this root represents a genuine second solution, its series expansion converges very slowly up to $x=1$.

VI. The following problem produced several known solutions.

$$
u^{\prime \prime}=2 u^{\prime}\left(u^{\prime}+1\right) /(u-x), \quad u(0)=1, \quad u(1)=0 .
$$

The solutions are

$$
u=1-x \text { and } u=\mathrm{c}(x-1) /(x-c), \quad c \neq 0 .
$$

All the approximate solutions were calculated with $\eta_{0}=-.75$.

The approximation of degree 19 produced three real roots:

$$
\eta=0,-.25,-.5 \text {. }
$$

The root -.25 produced the exact expansion $u=1-x$. The others corresponded to the second solution, $c= \pm 4.0$.

Recalculation of the solution using higher degree polynomials produced several other values for $c, \eta$. These values did not appear to be converging to any limit. The first solution $u=1-x$ was not obtained again. 
The most serious restriction of this method is that it is based on generating a Taylor series in two variables. It may be that the series does not converge in one or both variables in a sufficiently large domain. The Cauchy-Kowalewski theorem only guarantees convergence "in the small." This difficulty was encountered in most of the examples tried. But, in spite of this restriction, many problems were successfully solved.

The advantage of the method is that it may produce several solutions simultaneously. There is no guarantee that all the real solutions will be produced. Similarly, each real root of (6) may not correspond to a convergent solution. However, nonlinear differential equations lacking uniqueness are not at all unusual, so there exists a need for a technique that will produce several solutions when more than one solution exists. Only the physics of the problem can determine which solution of solutions are reasonable.

These examples indicate that the method can be fast and accurate. All the calculations were performed on a CDC 1604, which is a 10-place machine.

The author is greatly indebted to Louis Bushard and Michael Skow who chose and programmed many of these examples.

Tables. The table numbers correspond to the example numbers above. The entries of Tables I, II, IIIa, IVa, and V relate the true and approximate function values given on the interval $(0,1)$ in steps of .2. The entries of Tables IIIb and IVb are the values given in the interval $(0,1)$ with steps of -.2 starting at $x=1$. The true solution is indicated above the first column of each table. In those tables where there was more than one additional column, the value of the first derivative is indicated.

TABLE I

\begin{tabular}{cc}
\hline$u(x)=e^{2}+e^{-1}$ & $u(x)-U(x)$ \\
\hline $2.000,000,000$ & $10^{-10}$ \\
$2.310,555,451$ & $10^{-10}$ \\
$2.895,860,975$ & $10^{-10}$ \\
$3.868,928,559$ & $1 \times 10^{-9}$ \\
$5.402,361,389$ & $2 \times 10^{-9}$ \\
$7.756,935,541$ & $1 \times 10^{-9}$ \\
\hline
\end{tabular}

TABLE II

\begin{tabular}{cccc}
\hline$u(x)=2 /(2-x)$ & $\begin{array}{c}u(x)-U(x) \\
(.500,000,000,8)\end{array}$ & $\begin{array}{c}U(x) \\
(-10.65)\end{array}$ & $\begin{array}{c}U(x) \\
(-99.72)\end{array}$ \\
\hline $1.000,000,000$ & $10^{-10}$ & 1.000 & 1.0 \\
$1.111,111,111$ & $10^{-10}$ & -1.189 & $-1.2 \times 10^{1}$ \\
$1.250,000,000$ & $10^{-10}$ & -2.941 & $1.2 \times 10^{8}$ \\
$1.428,571,429$ & $10^{-10}$ & -3.989 & $7.8 \times 10^{12}$ \\
$1.666,666,667$ & $1 \times 10^{-9}$ & -100.4 & $1.3 \times 10^{16}$ \\
$2.000,000,000$ & $10^{-10}$ & +2.000 & $-5.3 \times 10^{9}$ \\
\hline
\end{tabular}


TABLE III

(a)

$$
u(x)=1+\log (1+x) \quad u(x)-U(x)
$$

(b)

$\begin{array}{ccr}10^{-4} & 1.693,147,181 & 10^{-10} \\ 2 \times 10^{-3} & 1.587,786,665 & 10^{-10} \\ 4 \times 10^{-3} & 1.470,003,629 & 10^{-10} \\ 1 \times 10^{-3} & 1.336,472,237 & 1 \times 10^{-9} \\ 7 \times 10^{-3} & 1.182,321,557 & 10^{-10} \\ 10^{-4} & 1.000,000,000 & 1 \times 10^{-9}\end{array}$

b)

$u(x)$ $u(x)-U(x)$

TABLE IV

(a)

\begin{tabular}{cccc}
$u(x)=(1+x)^{1 / 2}$ & $u(x)-U(x)$ & $u(x)$ & $u(x)-U(x)$ \\
\hline $1.000,0$ & $10^{-5}$ & $1.414,213,562$ & $10^{-10}$ \\
$1.095,4$ & $1 \times 10^{-4}$ & $1.341,640,786$ & $10^{-10}$ \\
$1.183,2$ & $2 \times 10^{-4}$ & $1.269,911,064$ & $10^{-10}$ \\
$1.264,9$ & $2 \times 10^{-3}$ & $1.183,215,957$ & $10^{-10}$ \\
$1.341,6$ & $4 \times 10^{-4}$ & $1.095,445,115$ & $10^{-10}$ \\
$1.414,2$ & $10^{-5}$ & $1.000,000,000$ & $1 \times 10^{-9}$ \\
\hline
\end{tabular}

1.000

1.182

1.336

1.470

1.588

1.693

TABLE V

\begin{tabular}{lrrrr}
\hline \hline$u(x)=(2+x) /(2-x)$ & $\begin{array}{c}u(x)-U(x) \\
(1.000,005,557)\end{array}$ & $\begin{array}{c}U(x) \\
(.095)\end{array}$ & $\begin{array}{c}U(x) \\
(8.68)\end{array}$ & \multicolumn{1}{c}{$\begin{array}{c}(45) \\
\end{array}$} \\
\hline $1.000,000,000$ & $10^{-10}$ & 1.000 & 1.000 & 1.000 \\
$1.222,222,222$ & $-7.8 \times 10^{-7}$ & 1.093 & 1.186 & 5.700 \\
$1.500,000,000$ & $-9.8 \times 10^{-7}$ & 1.333 & 2.474 & $2.262 \times 10^{9}$ \\
$1.857,142,857$ & $-7.7 \times 10^{-7}$ & 1.721 & $-2.070 \times 10^{3}$ & $5.016 \times 10^{14}$ \\
$2.333,333,333$ & $-4.2 \times 10^{-7}$ & 2.259 & $-9.414 \times 10^{6}$ & $1.990 \times 10^{18}$ \\
$3.000,000,000$ & $3.7 \times 10^{-8}$ & 3.000 & $-1.120 \times 10^{2}$ & $-5.773 \times 10^{12}$ \\
\hline
\end{tabular}

Appendix. In this section two examples are presented to illustrate how the algorithms in [4] are used to solve two point boundary value problems.

We shall use algorithms which give the expansions of products, ratios, and exponentials of polynomials in two variables. Let $P(I, J)$ denote the coefficient of $x^{I-1} y^{J-1}$ in the polynomial $\sum_{I-1}^{N} \sum_{J=1}^{N} P(I, J) x^{I-1} y^{J-1}$. The formulas for multiplication and division are as follows.

If

$$
R(x, y)=P(x, y) Q(x, y)
$$

then

$$
R(I, J)=\sum_{L=1}^{I} \sum_{M=1}^{J} P(L, M) Q(I-L+1, J-M+1) .
$$


If

$$
R(x, y)=P(x, y) / Q(x, y)
$$

then

$$
\begin{aligned}
R(I, J)=\frac{1}{Q(1,1)}\left(P(I, J)-\sum_{K=1}^{I-1} \sum_{L=1}^{J} R(K, L) Q(I-\right. & +1, J-L+1) \\
& \left.-\sum_{L=1}^{J-1} R(I, L) Q(1, J-L+1)\right) .
\end{aligned}
$$

The formula for exponentiation follows.

Let

then

$$
Q(x, y)=\exp (P(x, y))
$$

$$
\begin{aligned}
Q(1,1) & =\exp (P(1,1)) \\
Q(1, J+1) & =(1 / J) \sum_{L=0}^{J-1}(L+1) P(1, L+2) Q(1, J-L), \quad J=1, \cdots, N-1 \\
Q(I+1, J) & =(1 / I) \sum_{K=0}^{I-1} \sum_{L=0}^{J-1}(K+1) P(K+2, L+1) Q(I-K, J-1), \\
I=1, \cdots, N-1, \quad J=1, \cdots, N . &
\end{aligned}
$$

Let us consider the equation

$$
u^{\prime \prime} u+\left(u^{\prime}\right)^{2}=0, \quad u(0)=1, \quad u(1)=2^{1 / 2} .
$$

Set

and

$$
P(x, y)=u, \quad P(0, y)=1
$$

$$
\begin{aligned}
P_{x}(x, y) & =Q(x, y), \quad Q(0, y)=y, \\
R(x, y) & =-Q^{2}(x, y), \\
S(x, y) & =R(x, y) / P(x, y), \\
Q_{x}(x, y) & =S(x, y) .
\end{aligned}
$$

The initial data imply

$$
P(1,1)=Q(1,2)=1, \quad Q(1,1)=P(1, J)=Q(1, J+1)=0, \quad J \geq 2 .
$$

The remaining coefficients are determined by

$$
\begin{aligned}
P(I+1, J) & =Q(I, J) / I, \\
R(I, J) & =-\sum_{L=1}^{I} \sum_{M=1}^{J} Q(L, M) Q(I-L+1, J-M+1), \\
S(I, J)= & \frac{1}{P(1,1)}\left(R(I, J)-\sum_{K=1}^{I-1} \sum_{L=1}^{J} S(K, L) P(I-K+1, J-L+1)\right. \\
& \left.\quad-\sum_{L=1}^{J-1} S(I, L) P(1, J-L+1)\right),
\end{aligned}
$$


$Q(I+1, J)=S(I, J) / I, \quad J=1,2, \cdots, N$ for $I=1,2, \cdots, N-1$.

The values of the first derivative $y$ are then determined by the roots of

$$
\sum_{J=1}^{N} P_{J} y^{J-1}=2^{1 / 2} \text { where } P_{J}=\sum_{I=1}^{N} P(I, J) .
$$

As a second example let us consider the equation

$$
u^{\prime \prime}=u e^{u}, \quad u(0)=1, \quad u(1)=2 .
$$

Set

$$
P(x, y)=u, \quad P(0, y)=1,
$$

and

$$
\begin{aligned}
P_{x}(x, y) & =Q(x, y), \quad Q(0, y)=y, \\
R(x, y) & =\exp (P(x, y)), \\
S(x, y) & =P(x, y) R(x, y), \\
Q_{x}(x, y) & =S(x, y) .
\end{aligned}
$$

$P(1, J)$ and $Q(1, J)$ are the same as $P(1, J)$ and $Q(1, J)$ in the previous example.

and

$$
R(1,1)=\exp (P(1,1))
$$

$R(1, J+1)=(1 / J) \sum_{L=0}^{J-1}(L+1) P(1, L+2) R(1, J-L), \quad J=1,2, \cdots, N-1$.

The other coefficients are found by

$$
\begin{aligned}
P(I+1, J) & =Q(I, J) / I, \\
R(I+1, J) & =(1 / I) \sum_{K=0}^{I-1} \sum_{L=0}^{J-1}(K+1) P(K+2, L+1) R(I-K, J-L), \\
S(I, J) & =\sum_{L=1}^{I} \sum_{M=1}^{J} P(L, M) R(I-L+1, J-M+1), \\
Q(I+1, J) & =S(I, J) / I, \quad J=1,2, \cdots, N \quad \text { for } I=1,2, \cdots, N-1 .
\end{aligned}
$$

The solution is then found from

$$
\sum_{J=1}^{N} P_{J} y^{J-1}=2 \text { where } P_{J}=\sum_{I=1}^{N} P(I, J) .
$$

In both these examples the approximation to $U(x)$ is given by

where

$$
\sum_{I=1}^{N} C_{I} x^{I-1}
$$

$$
C_{I}=\sum_{J=1}^{x} P(I, J) y_{K}^{J-1},
$$

and the $y_{k}$ are determined by the polynomial equations. 


\section{REFERENCES}

[1] R. D. Richtmyer, Detached-shock calculations by power series. I, A. E. C. Research and Development Report, NYU-7973, New York University, Courant Institute of Mathematical Sciences, 1957

[2] G. Lewis, Two methods using power series for solving analytic initial value problems, A. E. C. Research and Development Report, NYO-2881, New York University, Courant Institute of Mathematical Sciences, 1960

[3] J. A. Leavitt, A power series solution for compressible flow past a conical shock wave, A. E. C. Research and Development Report, NYO-10, 432, New York University, Courant Institute of Mathematical Sciences, 1963

[4] J. A. Leavitt, Methods and applications of power series, Math of Comp. 20, January (1966)

[5] E. A. Coddington and N. Levinson, Theory of ordinary differential equations, McGraw-Hill, New York, 1955 